\title{
Development of Double Belt Type LC/MS Interface without Memory Effect
}

\author{
Tokuo Mizuno, Koushi Azuma and Kiichiro Otsuka \\ JEOL Ltd., Musashino, Akishima, Tokyo 196
}

\begin{abstract}
A new moving belt type LC/MS interface (ITF) combining with fast atom bombardment (FAB) ionization has been developed. Unlike the conventional endless belt type ITF, this ITF employs a disposable belt. Furthermore, this incorporates a double belt structure that covers the sample surface with another belt when passing through the differentially pumped region. The performance of LC/MS with this ITF was investigated. This ITF has completely eliminated the problems of memory effect so far encountered. By applying this ITF to the analysis of cyclodextrin mixture, chromatograms and mass spectra based on FAB ionization were obtained.
\end{abstract}

Keywords Moving belt LC/MS interface, disposable belt, double belt, memory effect, cyclodextrin

Development of efficient LC/MS interfaces (ITF) has been attempted by many people for a long period of time, with the result that various types of ITFs have been commercialized. ${ }^{1,2}$

Among the LC/MS ITFs developed in early stages of their technology is included an ITF that is called the "moving belt" method, where the effluent from LC is transferred on a moving belt and, after removing the solvent, the sample components on the belt are carried into an ion source for ionization. ${ }^{3}$

As for this moving belt type ITF, solvent removal and sample ionization are carried out at independent sections to prevent the solvent from flowing into the ion source. Therefore, it allows the use of an ion source in a relatively clean state as well as the use of various ionization methods such as EI, CI and FAB.,5

With the conventional moving belt ITF, however, the memory effect has been pointed out as a serious problem which is inevitably encountered when an endless belt is used repeatedly. ${ }^{3,6,7}$

One of the reasons for the memory effect is associated with the fact that an about one-meter-long endless belt is used repeatedly while cleaning it. Various cleaning methods have been attempted, including belt heating in a vacuum using a clean-up heater, belt washing with a circulating solvent under atmospheric pressure and forced removing of sample residue using a scrubber. ${ }^{3,8}$ A highly adhesive sample, however, cannot be completely removed from the belt, causing its remnant to bring about the memory effect.

Another cause of the memory effect is closely related to the fact that the belt ( $75 \mu \mathrm{m}$ thick and $3.17 \mathrm{~mm}$ wide) advances through a narrow tunnel seal $(150 \mu \mathrm{m}$ thick and $3.25 \mathrm{~mm}$ wide) in a differential pumping system. ${ }^{3}$ Slight vibrations of the belt and ruggedness of the belt surface sometimes cause the sample to stick to the inner surface of the tunnel seal. This produces a memory effect, which causes pseudo-peaks on chromatograms and/or the peak broadening.

Thus, in order to solve the biggest problem of the moving belt method, i.e., the memory effect, the following two countermeasures might be effective:

1) To use a clean endless belt

2) To prevent the belt surface coated with the sample from touching the inner wall of the narrow tunnel seal in the differential pumping system

Recently, DeMaio proposed the use of a disposable belt to eliminate the background caused by the memory effect. ${ }^{9}$ In this work, we have modified the disposable belt type ITF by employing a double belt structure, to avoid belt contamination when the belt passes through the tunnel seal. Such a belt-type ITF was incorporated to construct a new LC/MS system. The fundamental characteristics of the new system was studied in detail.

\section{Experimental}

Interface (ITF)

The block diagram of the interface developed is shown in Fig. 1. Two belts, sample belt (S-belt) and auxiliary belt (aux-belt) are fed from winders 1 and 2, respectively. These belts, driven by a drive motor $\left(\mathbf{M}_{1}\right.$ : UPH 266-A, Oriental Motor), advance between an atmospheric pressure section and a fast atom bombardment (FAB) ion source, via the tunnel seal $\left(t_{1}-t_{6}\right.$ : distance between seal surfaces $c a .200 \mu \mathrm{m}$ ) in the differential pumping system.

Differentially pumped chambers, $A_{1}$ and $A_{2}$, pumped by two dry pumps $(1201 / \mathrm{min}$ each) and two rotary 


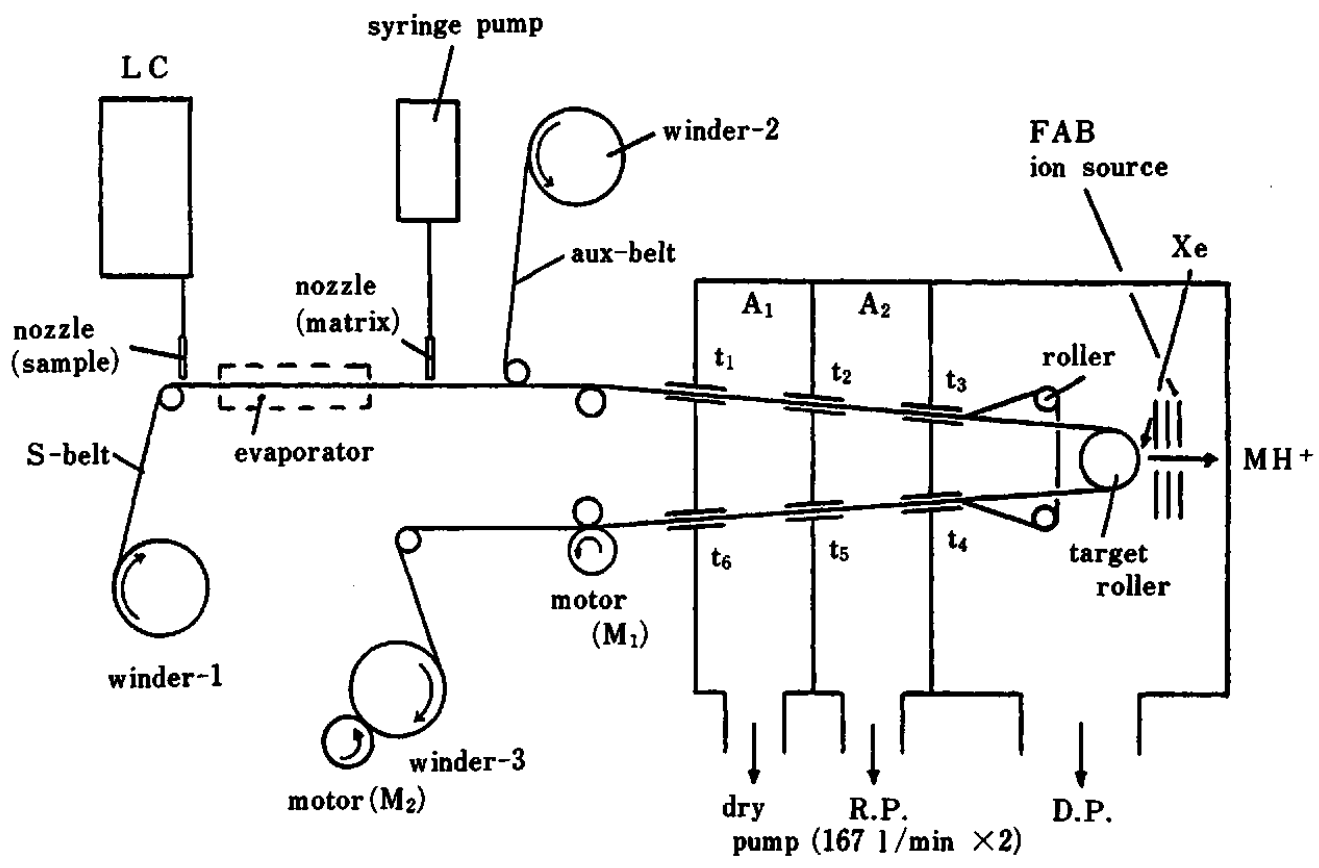

$(120 \mathrm{l} / \mathrm{min} \times 2)$

Fig. 1 Diagram showing the disposable/double belt LC/MS interface.

pumps ( $167 \mathrm{l} / \mathrm{min}$ each), are maintained at 80 Torr and 0.5 Torr, respectively. The effluent from $L C$ is transfered from a nozzle to the sample belt. After the solvent is removed by an evaporator (a pyrex glass tube, $3 \mathrm{~cm} \phi \times 20 \mathrm{~cm}, \max$. $300 \mathrm{~W}$ ), the matrix component (glycerol) is added using a syringe pump (FAMILIC-100N, Jasco). The sample on the belt is carried on a target roller in the FAB ion source and is ionized by a FAB beam.

Here the double belt structure enables the sample belt to be covered with an auxiliary belt only when passing through the seal section, to prevent belt contamination by the seal surfaces. The sample belt, after passing through the tunnel seal $\left(t_{3}\right)$, is separated from the auxiliary belt, sent in a different direction and bombarded by fast neutral particles, while the auxiliary belt is turned by rollers outside the FAB ion source, as shown in the figure. Both belts are again put together at the tunnel seal $t_{4}$ and, after passing through the tunnel seal $t_{6}$, are wound by winder -3 driven by $a$ synchronous motor $\mathbf{M}_{2}$.

\section{Belt}

The trially manufactured belt is $75 \mu \mathrm{m}$ thick, $6 \mathrm{~mm}$ wide, and $100 \mathrm{~m}$ long, and is made of a commercial polyimide film (Kapton). In order to increase the wettability of the belt surface with the mobile phase solvent, only one of the two surfaces was subjected to sand mat processing to give it ruggedness of 2 to 10 $\mu \mathrm{m}$ (Fig. 2(A)). With this processing, it has become possible to apply continuously even a polar solvent $\left(\mathrm{H}_{2} \mathrm{O} 100 \%\right)$ to the processed surface.

With the auxiliary belt, on the other hand, the unprocessed surface (B) was used as the contact surface with the sample. Using these two belts, glycerol was applied to the sample belt sequentially using a syringe pump (Fig. 1).

After the sample and the auxiliary belts were separated from each other at the tunnel seal $\left(t_{3}\right)$, the glycerol components remaining on each belt of unit length were weighed on a balance. The glycerol amounts on the belts were in the ratio $7: 3$. This indicates that, if the sample and glycerol were mixed uniformly, about $70 \%$ of the sample would remain on the sample belt.

\section{Instrument and measurement conditions}

The liquid chromatograph used for the experiment was FAMILIC-300S (Jasco). The columns used were $\mu$ s-Finepak SIL $\mathrm{C}_{18}$ and $\mu$ s-Finepak SIL $\mathrm{NH}_{2}$ $(1.5 \mathrm{~mm} \phi \times 25 \mathrm{~cm})$. The mobile phases used were $\mathrm{CH}_{3} \mathrm{CN}: \mathrm{H}_{2} \mathrm{O}(70 / 30)$ and $\mathrm{CH}_{3} \mathrm{CN}: \mathrm{H}_{2} \mathrm{O}(60 / 40)[0.01 \%$ $\mathrm{NaCl}$ added]. Measurements were made at a flow rate of $100 \mu \mathrm{l} / \mathrm{min}$.

All mobile phase solvents were completely eliminated in the evaporator where the temperature is maintained at $\left(\mathrm{ca} .120^{\circ} \mathrm{C}\right.$ ). After removal of the solvent, glycerol was applied as the matrix on a dried sample at a flow rate of $1 \mu \mathrm{l} / \mathrm{min}$. The belt was driven in $0.1 \mathrm{~mm}$ steps at a speed of $0.25 \mathrm{~cm} / \mathrm{s}$.

The mass spectrometer used was the JMS-DX303 (JEOL). For data measurement and processing, the JMA-DA5000 (JEOL) was used. The gun voltage of the FAB ion source was $4 \mathrm{kV}$, emission current $10 \mathrm{~mA}$, ion accelerating voltage of the mass spectrometer $3 \mathrm{kV}$, and secondary electron multiplier voltage $1.4 \mathrm{kV}$. 


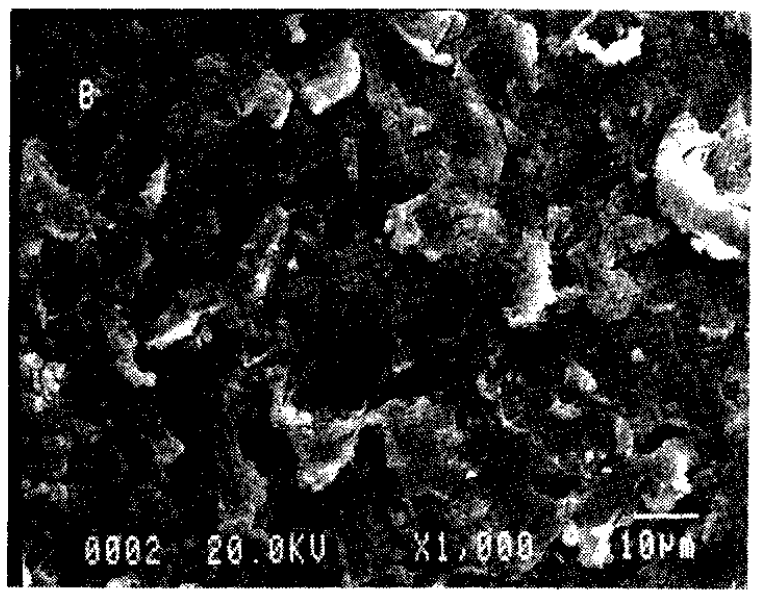

(A) Processed surface

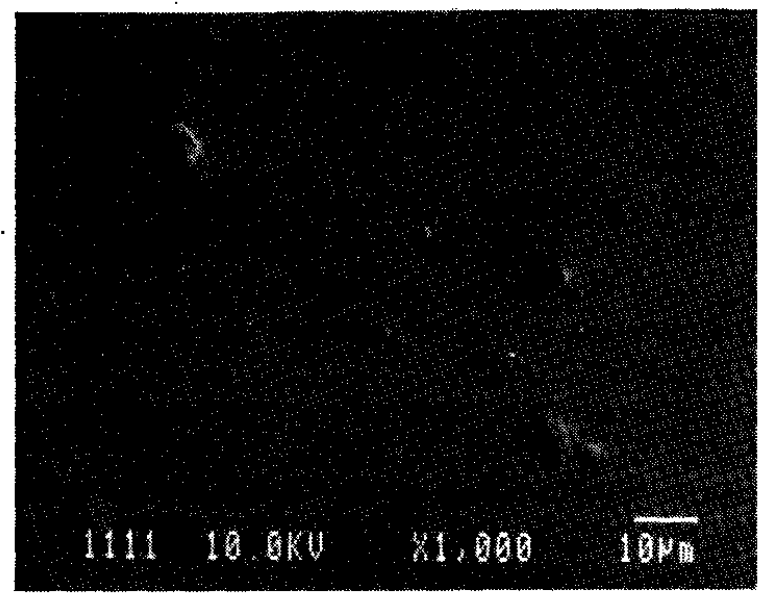

(B) Unprocessed surface

Fig. 2 Surface condition of the belt observed using electron microscope.

\section{Results and Discussion}

\section{Effect of auxiliary belt}

In order to know the effect of the auxiliary belt, vitamine $\mathrm{B}_{12}(1 \mu \mathrm{g})$ dissolved in $\mathrm{CH}_{3} \mathrm{CN}: \mathrm{H}_{2} \mathrm{O}(50 / 50)$ was applied, using a microsyringe, directly to three areas $[2 \mathrm{~cm}(L) \times 2 \mathrm{~mm}(W)]$ on the belt. These are located at intervals of about $4 \mathrm{~cm}$ and their mass fragmentograms were measured by driving the belt at a speed of $0.25 \mathrm{~cm} / \mathrm{s}$.

As shown in Fig. 3, when the auxiliary belt was not used, three peaks were not detected separately, and sample peaks also appeared from portions to which no sample was applied (Fig. 3(a)). Besides, sample peaks were sometimes detected $10 \mathrm{~min}$ or more later. This memory effect is mostly attributable to the direct contact of the sample belt to the tunnel seal surface despite a $100 \mu \mathrm{m}$ gap.

When the auxiliary belt is used, on the other hand, a mass fragmentogram having three $8 \mathrm{~s}$ wide peaks at
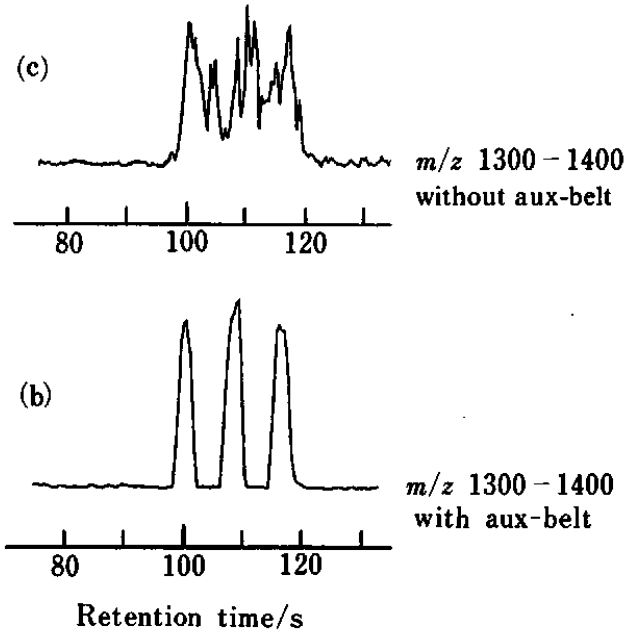

Fig. 3 Effect of aux-belt. Vitamin $B_{12}$ (M.W.1355, $1 \mu \mathrm{g}$ ) was deposited directly on the belt $(2 \mathrm{~cm}$ length, $4 \mathrm{~cm}$ interval). Glycerol, $1 \mu \mathrm{l} / \mathrm{min}$; belt speed, $0.25 \mathrm{~cm} / \mathrm{s}$.

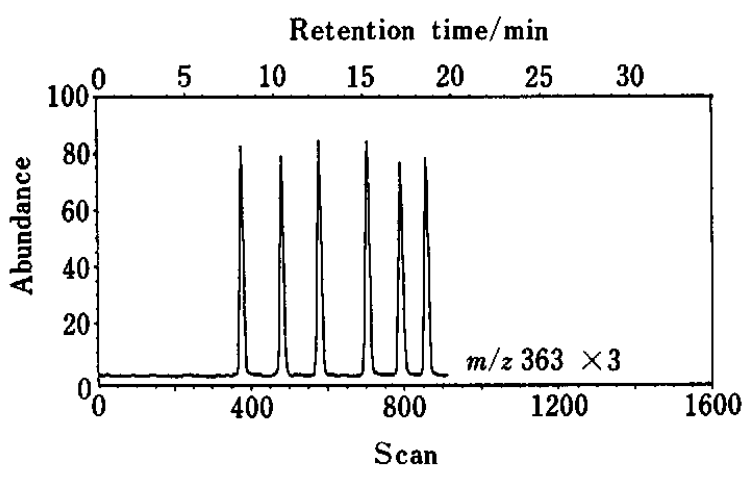

Fig. 4 Reproducibility of sample injections. Sample, cortisol $1 \mu \mathrm{g}$; column, $\mu \mathrm{s}$-Finepak SIL $\mathrm{C}_{18}(1.5 \mathrm{~mm} \phi \times 25 \mathrm{~cm})$; mobile phase, $\mathrm{CH}_{3} \mathrm{CN}: \mathrm{H}_{2} \mathrm{O}(70 / 30), 100 \mu \mathrm{l} / \mathrm{min}$.

$16 \mathrm{~s}$ intervals was obtained (Fig. 3(b)). In this case, the observed peak width agreed with that calculated from the sample application interval and width, and the belt speed $(0.25 \mathrm{~cm} / \mathrm{s})$.

These data indicate that the memory at the tunnel seal section, as with the conventional endless belt, was completely eliminated for the first time by the installation of the auxiliary belt.

\section{Basic performance of ITF}

By introducing a sample from LC, the basic performance (reproducibility, sensitivity, and resolution) of the LC/MS system was investigated.

1) Reproducibility

Figure 4 shows a chromatogram $\left(\mathrm{MH}^{+} 363\right)$ obtained by repeatedly injecting $1 \mu \mathrm{g}$ of cortisol (M.W. 362) at time intervals of about $2 \mathrm{~min}$. The resulting chromatogram consists of sharp and symmetric peaks and the base line is also stable. 
In addition, since mat processing has made it possible to apply the sample uniformly on the belt surface, spike-like noise ${ }^{10}$, which has so far posed a problem, was not observed. The reproducibility of the chromatogram peak intensity was $4 \%$ (relative standard deviation value) for six runs.

2) Resolution

In order to know whether the chromatographic resolution is maintained or not, measurement of $1 \mu \mathrm{g}$ of cortisol was conducted under the same conditions mentioned above by connecting a UV detector (UVIDEC-100IV) between the column and ITF (Fig. 5).

The upper trace is a UV chromatogram measured at $\lambda=250 \mathrm{~nm}$, and the lower trace is a mass chromatogram obtained with $\mathrm{MH}^{+}(m / z$ 363). The mass chromatographic peaks were detected at a time lag of about $4 \mathrm{~min}$. This is a reasonable value, considering the belt speed $(0.25 \mathrm{~cm} / \mathrm{s})$ and the distance between the sample dripping position and the ion source $(c a .60 \mathrm{~cm})$.

The facts that the peak width $\left(W^{1 / 2}\right)$ is about $15 \mathrm{~s}$ for both chromatograms and that no appreciable peak

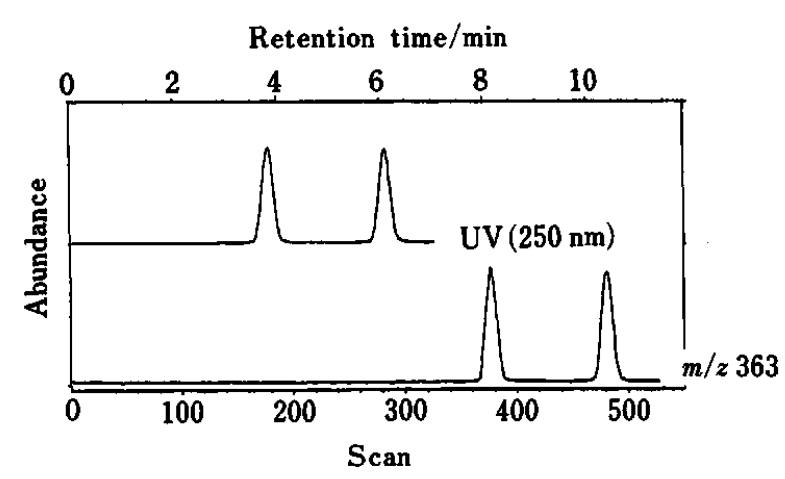

Fig. 5 Comparison between UV and mass chromatogram. Sample, cortisol $1 \mu \mathrm{g}$; column, $\mu \mathrm{s}$-Finepak SIL $\mathrm{C}_{18}$ $(1.5 \mathrm{~mm} \phi \times 25 \mathrm{~cm}) ;$ mobile phase, $\mathrm{CH}_{3} \mathrm{CN}: \mathrm{H}_{2} \mathrm{O}(70 / 30)$, $100 \mu \mathrm{l} / \mathrm{min}$.

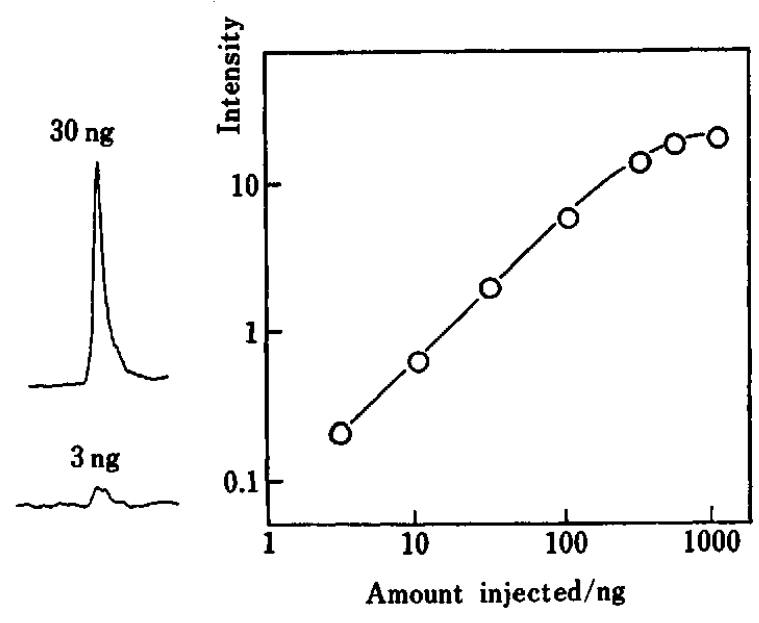

Fig. 6 SIM chromatograms of $(M+H)^{+}$ion and their intensity as a function of nanograms of cortisol. broadening is observed suggest that the LC resolution is not impaired even after passing through this ITF.

3) Sensitivity

Fan et al. measured $1.25 \mu \mathrm{g}$ to $25 \mu \mathrm{g}$ of histidine. ${ }^{11}$ Stroh et al. also reported a quantitative result for $125 \mathrm{ng}$ of peptide (Ac-Phe-Aib-Aib-Iva-OH), using an ITF of the same type and detected $(\mathrm{M}+\mathrm{Na})^{+}$ion with an $S / N$ ratio of $3 .{ }^{12}$ On the other hand, Gaskell $e t$ al. prepared calibration curves for 50 to $550 \mathrm{ng}$ of cortisol by means of thermospray (TSP). ${ }^{13}$

We measured $3 \mathrm{ng}$ to $1000 \mathrm{ng}$ samples of cortisol in the selected ion monitoring (SIM) mode under the same analytical conditions as mentioned above (Fig. 6). As a result, an SIM chromatogram $\left(\mathrm{MH}^{+} m / z\right.$ 363) of $3 \mathrm{ng}$ injection of cortisol was obtained with high sensitivity, $S / N 5$. It was also known that a linear relationship was obtained between $3 \mathrm{ng}$ to $100 \mathrm{ng}$. The sensitivity indicated by the above result is almost equivalent to that for bile acids obtained using the ordinary FAB probe. ${ }^{14}$

\section{Application}

The newly developed ITF was applied to the analysis of a cyclodextrin mixture ( $\alpha-$ : M.W. $972 \beta-:$ M.W. 1134, $\gamma$-: M.W. 1296, $5 \mu \mathrm{g}$ each). The analysis was conducted using an amino column ( $\mu$ s-Finepak SIL $\mathrm{NH}_{2}: 1.5 \mathrm{~mm} \phi \times 25 \mathrm{~cm}$ ).

In the case of cyclodextrins, the sensitivity of molecular ions $\left(\mathrm{MH}^{+}\right)$is relatively low with FAB. Therefore, the cyclodextrins were analyzed by adding $0.01 \%$ of $\mathrm{NaCl}$ to the mobile phase $\left[\mathrm{CH}_{3} \mathrm{CN}: \mathrm{H}_{2} \mathrm{O}\right.$ $(60 / 40)]$ and setting the flow rate to $100 \mu \mathrm{l} / \mathrm{min}$. Mass chromatograms due to $(\mathrm{M}+\mathrm{Na})^{+}$ions of the three components and a mass spectrum of $\alpha$-cyclodextrin are shown in Fig. 7 (a) and (b), respectively. The three components were clearly separated and stably detected.

The mass spectrum shows weak $(\mathrm{M}+\mathrm{H})^{+}(m / z$ 973) ions, and cluster ions to which $\mathrm{Na}$ was added, $(\mathrm{M}+\mathrm{Na})^{+}(\mathrm{m} / \mathrm{z} 995)$, were detected as the base peak. Incidentally, the peak intensity of $(\mathrm{M}+\mathrm{Na})^{+}$ions of the three components increased to about 3 to 4 times that of $(\mathrm{M}+\mathrm{H})^{+}$ions obtained without addition of salt. But when the concentration of salt was increased to $0.05 \%$, the intensity of $(\mathrm{M}+\mathrm{Na})^{+}$ions showed a tendency to decrease.

With the conventional LC/MS system, the use of inorganic salt is virtually impossible. Therefore it has been necessary to establish the analytical conditions for an organic buffer system, prior to making analysis.6,15 With our ITF, in which unnecessary salt is constantly carried away from the instrument via the belt, the salt is not accumulated in the ionization chamber and/or on the belt. Therefore, in this LC/MS system, it is possible to use inorganic buffers. Consequently, the application scope of our new LC/MS system would be markedly wider than the conventional ones.

A newly-developed disposable double-belt-type ITF has completely eliminated the problem of the conven- 


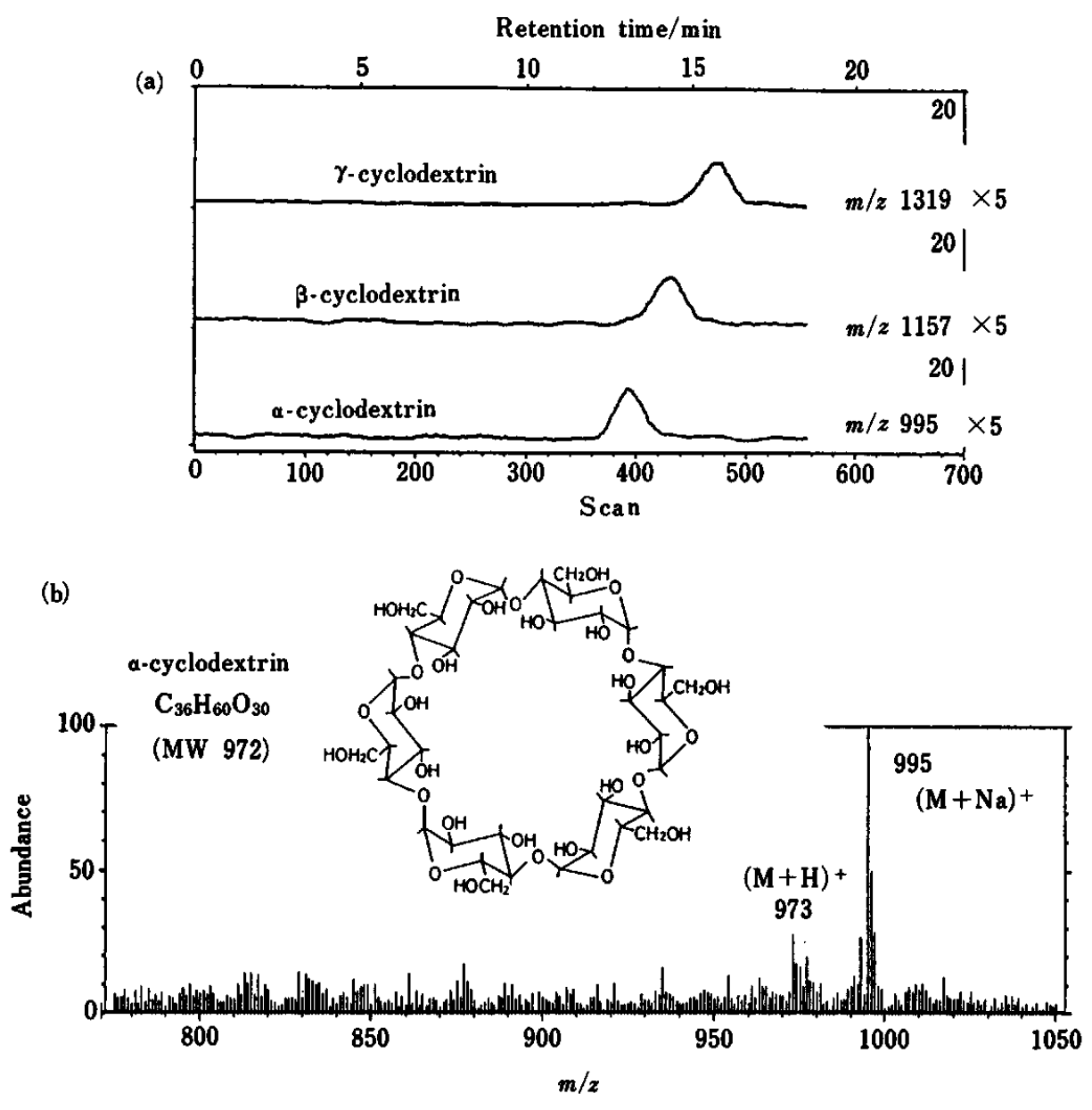

Fig. 7 Mass chromatograms of cyclodextrin mixture (a) and mass spectrum of $\alpha$-cyclodextrin (b). M.W.: $\alpha$-, 972; $\beta$-, $1134 ; \gamma$-, 1296, each $5 \mu$ g; column, $\mu$ s-Finepak SIL $\mathrm{NH}_{2}(1.5 \mathrm{~mm} \phi \times 25 \mathrm{~cm})$; mobile phase, $\mathrm{CH}_{3} \mathrm{CN}: \mathrm{H}_{2} \mathrm{O}(60 / 40), 0.1 \% \mathrm{NaCl}, 100$ $\mu \mathrm{l} / \mathrm{min}$.

tional moving belt type ITF, namely, the memory effect caused by repeated use of the belt and by the tunnel seal section. At the same time, the new ITF has eliminated the need of a complicated belt-washing mechanism.

Sample measurement with this ITF has proved that, although the use of FAB ionization puts some limitation to the linearlity range in sensitivity, reproducible chromatograms could be obtained and that $L C$ resolution was not impaired.

Also, as shown by the analytical results of cyclodextrins, addition of inorganic salt, which has so far been regarded as difficult, has now become possible with the adoption of this disposable belt. These results suggest that our ITF, which utilizes the FAB ionization method, would be fully usable for the analysis of various nonvolatile or thermally labile compounds.

The belt used for the experiment now costs about ten dollars for one roll $(100 \mathrm{~m})$ and when operated at a belt speed of $0.25 \mathrm{~cm} / \mathrm{s}$, can be continuously used for about $11 \mathrm{~h}$. Also, the belt can of course be effectively washed outside the ITF system for its repeated use.

The greatest feature of the belt-type ITF is its applicability to a wide range of ionization methods including not only FAB but also EI, CI, etc. 1,4,5 From now on, we plan to apply this newly developed disposable double-belt-type ITF to these ionization modes and to evaluate the results.

\section{References}

1. T. R. Covey, E. D. Lee, A. P. Bruins and J. D. Henion, Anal. Chem., 58, 1451A (1986).

2. P. J. Arpino and G. Guiochon, Anal. Chem., 51, 682A (1979).

3. W. H. McFadden and H. L. Schwartz, J. Chromatogr., 122, 389 (1976).

4. P. Dobberstein, E. Korte, G. Meyerhoff and R. Pesch, Int. J. Mass Spectrom. Ion Phys., 46, 185 (1983).

5. D. E. Games, Biomed. Mass Spectrom., 8, 454 (1981).

6. S. Tsuge, "Kagaku no Ryoiki Zokan" 132, 155, Nankodo (1981).

7. W. H. McFadden, J. Chromatogr. Sci., 18, 97 (1980).

8. R. D. Smith, J. E. Bruger and A. L. Johnson, Anal. Chem., 53, 1603 (1981).

9. W. DeMaio, Proceedings of 35th ASMS, p. 423 (1987), Denver. 
10. M. J. Hayes, E. P. Lankmayer, P. Vouros and B. L. Karger, Anal. Chem., 55, 1745 (1983).

11. T. P. Fan, E. D. Hardin and M. L. Vestal, Anal. Chem., 56, 1870 (1984).

12. J. G. Stroh, J. C. Cook, R. M. Milberg, L. Brayton, T. Kihara, Z. Huang and K. L. Rinehart, Anal. Chem., 57, 985 (1985).
13. S. J. Gaskell, K. Rollins, R. W. Smith and C. E. Parkar, Biomed. Mass Spectrom., 14, 717 (1987).

14. K. Tanaka, GC-MS News, Vol. 12, No. 1, 4 (1984).

15. C. N. Kenyon, Biomed. Mass Spectrom., 10, 535 (1983).

(Received February 20, 1988) (Accepted April 16, 1988) 